\title{
Navigability Analysis of Natural Terrains with Fuzzy Elevation Maps from Ground-based 3D Range Scans
}

\author{
Jorge L. Martínez, Anthony Mandow, Antonio Reina, \\ Tomás J. Cantador, Jesús Morales and Alfonso García-Cerezo \\ Departamento de Ingeniería de Sistemas y Automática \\ University of Málaga, 29071 Málaga, Spain. Email: jlmartinez[@]uma.es
}

\begin{abstract}
Mobile robot navigation through natural terrains is a challenging issue with applications such as planetary exploration or search and rescue. This paper proposes navigability assessment of natural terrains scanned from groundbased 3D laser rangefinders. A continuous model of the terrain is obtained as a fuzzy elevation map (FEM). Based on this model, the proposed solution incorporates terrain navigability both in terms of uncertainties of the 3D input data and slope of the fuzzy surface. Moreover, the paper discusses the application of this method for local path planning. For this purpose, the Bug algorithm has been adapted to compute local paths on the navigable region of the FEM. The method has been applied to actual 3D point clouds on two different experimental sites.
\end{abstract}

Keywords: Mobile robots, terrain modelling, field robotics, 3D point clouds, laser scanner, path planning, robot control.

This document is a self-archiving copy of the accepted version of the paper.

Please find the final published version in: http://ieeexplore.ieee.org

\section{Citation Information}

Martínez, J. L.; Mandow, A.; Reina, A.; Cantador, T. J.; Morales, J.; García-Cerezo, A.; ,

"Navigability Analysis of Natural Terrains with Fuzzy Elevation Maps from Ground-based 3D Range Scans," IEEE/RSJ International Conference on Intelligent Robots and Systems (IROS), pp.1576-1581, Nov. 2013 doi:

@INPROCEEDINGS $\{$ Martinez: IROS2013,

author $=\{\mathrm{J}$. L. Mart $\backslash\{\mathbf{i}\}$ nez and A. Mandow and A. Reina and T. J. Cantador and J. Morales and A. Garc $\backslash\{i\} a-C e r e z o\}$, title $=\{$ Navigability Analysis of Natural Terrains with Fuzzy Elevation Maps from Ground-based 3D Range Scans $\}$, booktitle $=\{$ IEEE/RSJ International Conference on Intelligent Robots and Systems $\}$, year $=\{2013\}$

address $=\{$ Tokyo, Japan $\}$, pages $=\{1576-1581\}$

\}

(c) 2013 IEEE. Personal use of this material is permitted. Permission from IEEE must be obtained for all other uses, in any current or future media, including reprinting/republishing this material for advertising or promotional purposes, creating new collective works, for resale or redistribution to servers or lists, or reuse of any copyrighted component of this work in other works. 


\title{
Navigability Analysis of Natural Terrains with Fuzzy Elevation Maps from Ground-based 3D Range Scans
}

\author{
Jorge L. Martínez, Anthony Mandow, Antonio Reina, \\ Tomás J. Cantador, Jesús Morales and Alfonso García-Cerezo \\ Dpto. Ingeniería de Sistemas y Automática, Universidad de Málaga, 29071 Málaga, Spain \\ Email: jlmartinez@uma.es, Tel: (+34) 951952322.
}

\begin{abstract}
Mobile robot navigation through natural terrains is a challenging issue with applications such as planetary exploration or search and rescue. This paper proposes navigability assessment of natural terrains scanned from ground-based 3D laser rangefinders. A continuous model of the terrain is obtained as a fuzzy elevation map (FEM). Based on this model, the proposed solution incorporates terrain navigability both in terms of uncertainties of the 3D input data and slope of the fuzzy surface. Moreover, the paper discusses the application of this method for local path planning. For this purpose, the Bug algorithm has been adapted to compute local paths on the navigable region of the FEM. The method has been applied to actual 3D point clouds on two different experimental sites.
\end{abstract}

\section{INTRODUCTION}

Three-dimensional (3D) point clouds provide valuable information in mobile robotics applications such as planetary exploration [1] [2] [3] [4], urban search and rescue [5] [6], and navigation on natural terrain [7]. However, as point cloud maps require coping with a huge amount of spatial information [8] [9], a simplified and compact representation of navigable terrain is necessary for motion planning [1] or tele-operation [10].

Elevation maps offer a compact two dimensional model of terrain surface. In robotics, these maps have been represented as regular grids [11] [12] and as irregular triangular meshes [4] [13]. Removal of artifacts (i.e., triangles in concavities and sensor shadows) and mesh simplification algorithms, like JADE mesh decimation [14] and QSlim vertex clustering [15], provide more compact and reliable maps [1]. Nevertheless, tessellated models have limitations in the face of incomplete and uncertain sensor data, as well as in scalability. Alternatively, the use of Adaptive Neural-based Fuzzy Inference System (ANFIS) [16] was proposed in [17] to model natural terrain as a continuous Fuzzy Elevation Map (FEM).

For tessellated terrain representations, the path planning problem can be formulated as a graph-search problem to minimize a cost function that combines distance and terrain difficulty [4] [18]. Path planning in outdoors has also been addressed with Voronoi diagrams to maintain the robot far from non-traversable zones [19] and potential fields based on harmonic functions to avoid local minima [20].

Nevertheless, terrain assessment is a relevant problem that has to be solved prior to path planning and it is very dependent on the environment model. In outdoor environments, terrain difficulty has been quantified in terms of slope and roughness [4] [19]. Besides, a major difficulty arises from propagating uncertainties of $3 \mathrm{D}$ data to path planning [18] [21].

This paper proposes navigability assessment in natural terrains represented by FEMs obtained from ground-based 3D laser scans. The assessment captures both model reliability in terms of 3D data availability as well as terrain slope. The proposed processing of the FEM can be useful for local path planning, as illustrated with examples from actual 3D scans.

The paper is organized as follows. Next section reviews natural terrain modeling with FEMs. The extraction of the navigable area on FEMs is proposed in Section III. Section IV presents local path planning on assessed traversable terrain. Section V discusses experimental results. Last section is devoted to conclusions and future work.

\section{FUZZY ELEVATION MAPS}

This section briefly reviews the computation of a local FEM from a single range image taken from an onboard laser scanner [17]. The method, which is outlined in Fig. 1, assumes that:

- The local frame of the 3D rangefinder has its $Y$ and $Z$ axes pointing forwards and upwards, respectively.

- The ground surface can be represented as a function $z=$ $H(x, y)$, where $x$ and $y$ are the Cartesian coordinates on the $X Y$ plane and $z$ is the corresponding elevation.

- The FEM is defined in the universe $U_{x}=$ $\left[-u_{\max }, u_{\max }\right]$ for variable $x$, and $U_{y}=\left[0, u_{\max }\right]$ for $y$, which corresponds to a $2 u_{\max } \times u_{\max }$ rectangular area in the forward direction of the sensor.

The method uses ANFIS [16] to identify rule parameters from a set of training Cartesian points (see Fig. 1). Representative training points are subsampled from the raw scan by selecting the maximum height point within grid cells of a sufficiently high resolution $\delta$. In [17], first-order Sugeno inference was used, which requires identifying three consequent parameters per rule.

An uneven membership function distribution provides an appropriate fuzzy structure if the density of MFs for variables $x$ and $y$ is specified depending on the distance to the sensor. Thus, higher detail is captured for the regions that are closest to the robot where the next movements will take place. 


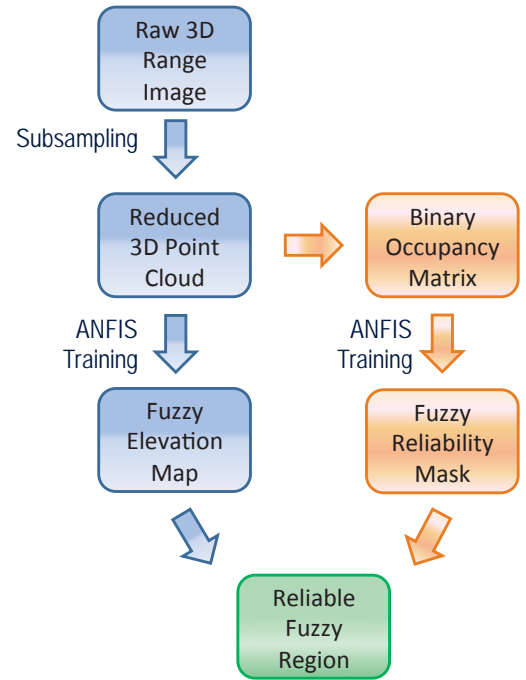

Fig. 1. Overview of the method to obtain a FEM from a raw scan [17].

The FEM filters sensor noise and interpolates missing data from small shadowed areas. However, it can provide completely erroneous estimations in larger regions with no input data. These shadowed regions are frequent in groundbased scans of natural terrain. To solve this problem, a fuzzy reliability mask was proposed as a continuous function $v=V(x, y)$, where $v \in[0,1]$ for inputs $x \in U_{x}$ and $y \in U_{y}$. For this purpose, an occupancy binary matrix is computed from the subsampled scan points. This matrix represents an $X Y$ grid with uniform resolution $\delta$, where ones and zeroes are assigned to positions corresponding to cells with or without points, respectively. Then, the reliability training data consists of the set of all matrix values with their corresponding $X Y$ coordinates (see Fig. 1). Regions with $v$ close to one mean that $H(x, y)$ is reliable and reliability decreases as $v$ approaches zero. This function can be trained with ANFIS with the same fuzzy structure as in the FEM. By applying the fuzzy reliability mask to the FEM, unreliable regions whose $v$ value is under a threshold value $v_{t}$ can be discarded.

\section{Navigable Area Assessment}

This section proposes processing the FEM to assess regions where terrain inclination is acceptable for vehicle navigation. The gradient of the FEM can be directly obtained without computing increments from sampled points. This analysis will be combined with the reliable fuzzy region to obtain the navigable area from a ground-based 3D scan.

For simplicity, let rule parameters be systematically defined by triangular sets with standard fuzzy partition (SPF) [22] as well as zero order Sugeno-type inference. SPF triangular MFs $\mathbf{F}_{i}$ for a given variable $u$ in the universe $U$ are defined as:

$$
\mu_{F_{i}}(u)= \begin{cases}\frac{u-f_{i-1}}{f_{i}-f_{i-1}} & \text { if } f_{i-1} \leq u<f_{i}, \\ \frac{f_{i+1}-u}{f_{i+1}-f_{i}} & \text { if } f_{i} \leq u<f_{i+1}, \\ 0 & \text { otherwise }\end{cases}
$$

where $f_{i}$ is the peak parameter, i.e., $\mu_{F_{i}}\left(f_{i}\right)=1$. In the proposed FEM solution, $i=-k, \ldots, 0,1, \ldots, k$ for the $x$ variable and $i=0,1, \ldots, k$ for $u=y$. This definition yields $(2 k+1)$ MFs for $x$ and $(k+1)$ MFs for $y$. Note that for the upper limit of $U$, where $f_{k}=u_{\max }$, the second case in (1) does not apply. Similarly, the first case in (1) does not apply for the lower limit of $U$.

Uneven SPF MFs are defined by computing the peak parameter $f_{i}$ as:

$$
f_{i}=\operatorname{sign}(i)\left(\frac{r^{|i|}-1}{r^{k}-1}\right) u_{\max },
$$

where $r>1$ is the expansion ratio.

Using zero-order Sugeno consequents $G_{i j}$ for the rule that relates $F_{i}(x)$ and $F_{j}(y)$ requires just one parameter $a_{i j}$ :

$$
G_{i j}(x, y)=a_{i j} .
$$

The firing strength of each rule $\omega_{i j}$ can be calculated using the product operator:

$$
\omega_{i j}(x, y)=\mu_{F_{i}}(x) \mu_{F_{j}}(y),
$$

where SPF MFs satisfy:

$$
\sum_{\forall i, j} \omega_{i j}(x, y)=1 .
$$

Thus, the elevation $z$ associated to $(x, y)$ can be calculated from the FEM as:

$$
z=H(x, y)=\sum_{\forall i, j}\left(\omega_{i j}(x, y) a_{i j}\right) .
$$

Then, the gradient $\nabla H$ for every $(x, y)$ can be directly calculated as:

$$
\nabla H(x, y)=\left(\begin{array}{c}
\frac{\partial z}{\partial x} \\
\frac{\partial z}{\partial y}
\end{array}\right)=\left(\begin{array}{c}
\sum_{\forall i, j}\left(\frac{\partial \mu_{F_{i}}(x)}{\partial x} \mu_{F_{j}}(y) a_{i j}\right) \\
\sum_{\forall i, j}\left(\mu_{F_{i}}(x) \frac{\partial \mu_{F_{j}}(y)}{\partial y} a_{i j}\right)
\end{array}\right),
$$

where:

$$
\frac{\partial \mu_{F_{i}}(u)}{\partial u}= \begin{cases}\frac{1}{f_{i}-f_{i-1}} & \text { if } f_{i-1} \leq u<f_{i}, \\ \overline{f_{i+1}-f_{i}} & \text { if } f_{i} \leq u<f_{i+1}, \\ 0 & \text { otherwise. }\end{cases}
$$

The value given by (7) is a representation of terrain inclination. Therefore, non traversable areas can be identified if their gradient magnitude

$$
|\nabla H(x, y)|=\sqrt{\left(\frac{\partial z}{\partial x}\right)^{2}+\left(\frac{\partial z}{\partial y}\right)^{2}},
$$

is above a threshold value $h$ that depends on the locomotion mechanism of the mobile robot. Even if the proposed assessment can be applied to the complete universe of discourse, the analysis can be restricted to the reliable regions of the FEM for computation efficiency. 


\section{Local Path Planning}

The proposed FEM navigability assessment can be useful for local path planning on natural terrain. This is illustrated in this section, where we propose a simple solution based on the well known Bug algorithm [23] to plan a continuous path towards a goal point by avoiding non traversable areas. An overview of the proposed approach is presented in Fig. 2.

In particular, the BugO algorithm [24] heads towards a goal point until it reaches an obstacle; then, the obstacle is circumnavigated until the goal point is visible again. This local path planning strategy is compatible with global paths composed of distant way points that are meant to be reached in straight line motion from the current robot pose [25] [26].

BugO requires computing a $2 \mathrm{D}$ binary representation of the environment that distinguishes between non navigable and navigable areas from the 2.5D FEM. Thus, non-navigable areas are treated as obstacles and the rest as free space. Furthermore, as the robot is not punctual, the Minkowski sum is applied to enlarge obstacles. The resulting binary representation may have unconnected free space zones that cannot be reached from the robot pose (i.e., $x=y=$ $0)$. Thus, only the reachable area will be considered for computing the local path.

Moreover, the goal point may fall beyond the range of the reachable area, so a subgoal has to be established. In the proposed solution, this subgoal is chosen as the point on the border of the reachable region that minimizes the distance to the global goal point. Then, the objective of $\mathrm{BugO}$ is heading towards this subgoal.

\section{EXPERIMENTAL RESUlts}

This section discusses the application of the proposed navigability assessment for local path planning. For this purpose, two natural terrains have been scanned (see Fig. 3) with a 3D rangefinder [27] built by pitching a Hokuyo UTM-30LX 2D rangefinder whose maximum range is $30 \mathrm{~m}$.

The laser sensor is mounted $0.7 \mathrm{~m}$ above the ground on the 4-wheel skid-steer mobile robot Quadriga [28] (see Fig. 4). Besides, Quadriga has an inertial measurement unit with

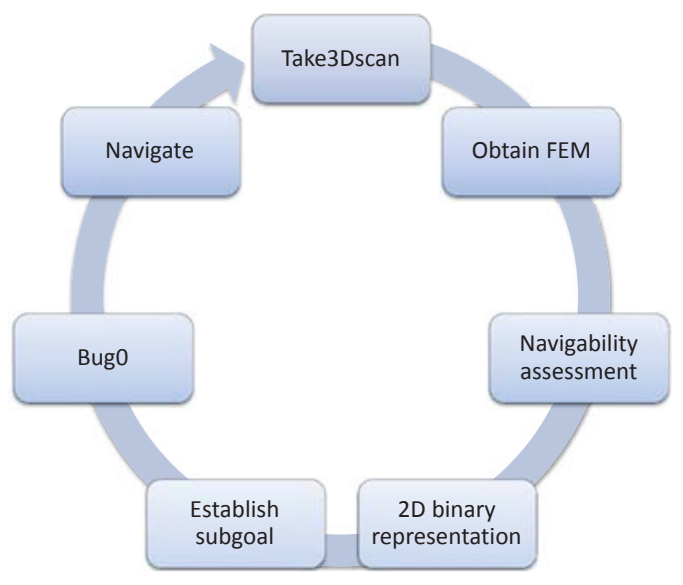

Fig. 2. Local path planning overview. inclinometers and a Global Positioning System with Differential corrections (DGPS). Inclinometers can be employed to check tipover stability during navigation. Furthermore, the DGPS can provide the distance and heading with respect to the next way point in outdoor missions.

The point clouds from both scans are shown in Fig. 5. The FEMs computed from these scans with $k=9, r=1.3$, $u_{\max }=10 \mathrm{~m}$ and $\delta=0.1 \mathrm{~m}$ have been overlaid on this figure, and as a top view in Figs. 6(a) and 7(a). Red and blue colors mean higher and lower elevations, respectively. In both cases, the complete terrain model is represented with only 190 rules and a total of 219 floating point parameters. These fuzzy surfaces contain artifacts with extreme elevation values in regions without training data, as those around the white patches in the top view.

The fuzzy reliability mask computed for both scans is shown in Figs. 6(b) and 7(b). Red and blue colors mean higher and lower reliability, respectively, according to the availability of training data. The application of the masks on their corresponding FEMs with a threshold of $v_{t}=0.1$ can be observed in Figs. 6(c) and 7(c). Discarded areas are represented in white.

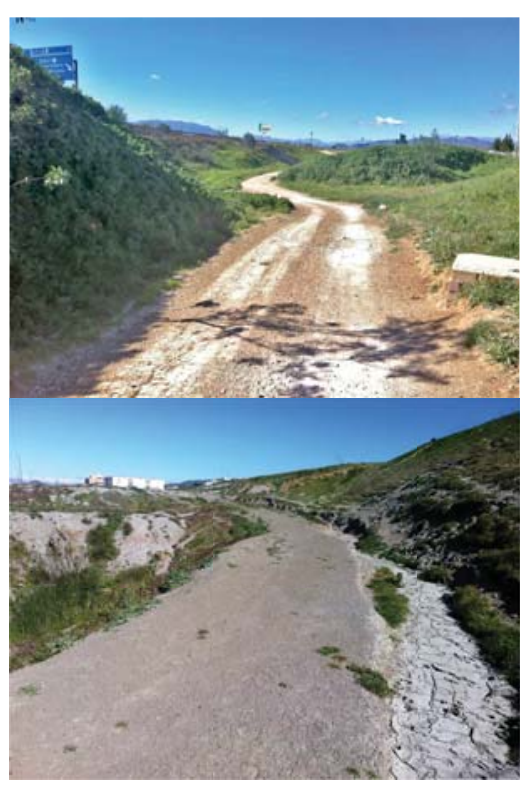

Fig. 3. The first (top) and second (bottom) experimental sites.

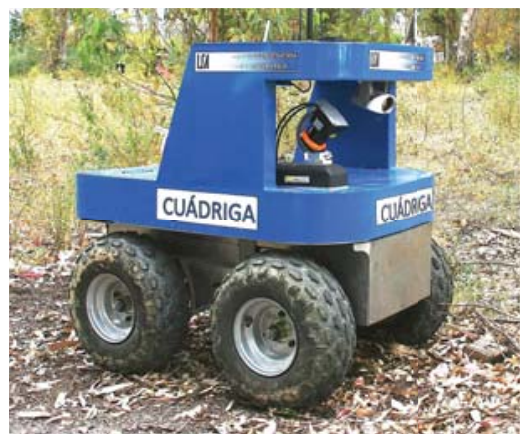

Fig. 4. Quadriga mobile robot with onboard 3D rangefinder. 

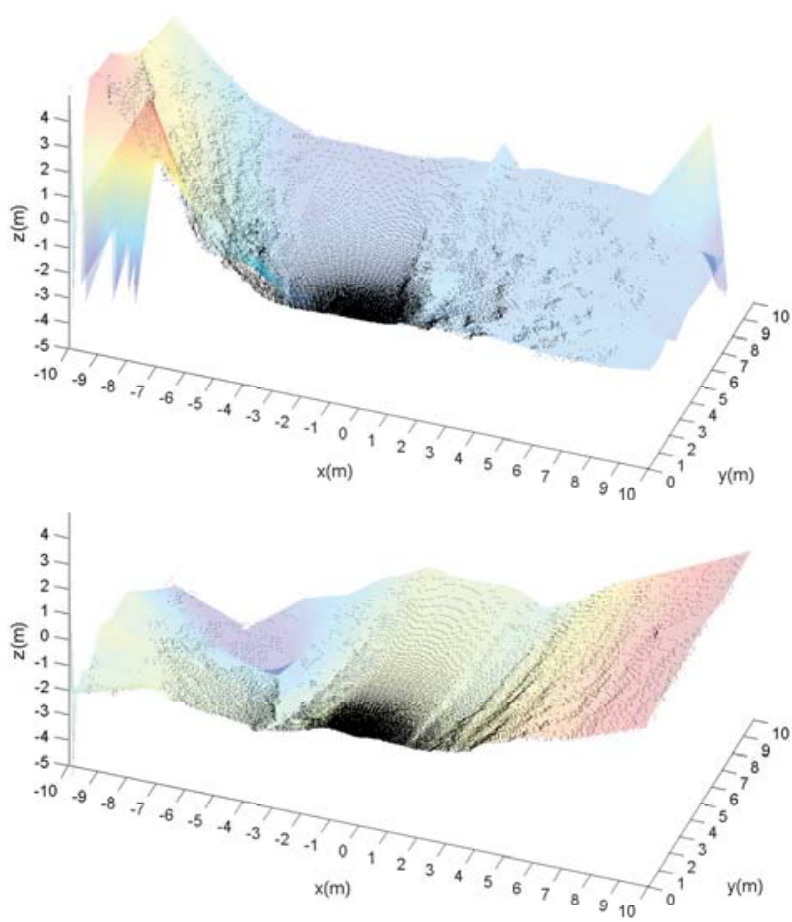

Fig. 5. Point clouds from the first (top) and second (bottom) scans and resulting FEMs.

The gradient magnitude $|\nabla H|$ computed with (9) for both scans is shown in Figs. 6(d) and 7(d). Red and blue colors mean higher and lower sloped regions, respectively. The application of a threshold of $h=0.5$, which corresponds to a maximum slope of $\arctan (h)=26.6^{\circ}$, can be observed in Figs. 6(e) and 7(e). Discarded areas are represented in white.

The navigable areas in the FEMs are shown in Figs. 8(a) and 9(a). The color difference between both figures is explained by general terrain inclination of the experimental sites, which is downwards (blue) in the first case and upwards (green) in the second.

The reachable regions for both FEMs after applying the Minkowski sum for the largest robot dimension and eliminating unconnected areas are shown in Figs. 8(b) and 9(b). Two different local path planning examples are illustrated in each figure with distant goal points at $\pm 30^{\circ}$ headings, which are shown as discontinuous red lines. The corresponding subgoals for BugO are denoted as 'o's. A 3D representation of the resulting paths on their corresponding FEMs is presented in Figs. 8(c) and 9(c).

\section{CONCLUSIONS AND FUTURE WORK}

This paper proposes navigable area assessment from fuzzy elevation maps (FEMs) of natural terrain obtained from a ground-based 3D laser scan. This solution incorporates terrain navigability both in terms of uncertainties of the 3D input data and slope of the fuzzy surface.

The proposed processing of the FEM can be useful for local path planning. For this purpose, the Bug algorithm has been adapted for obtaining local paths on the navigable

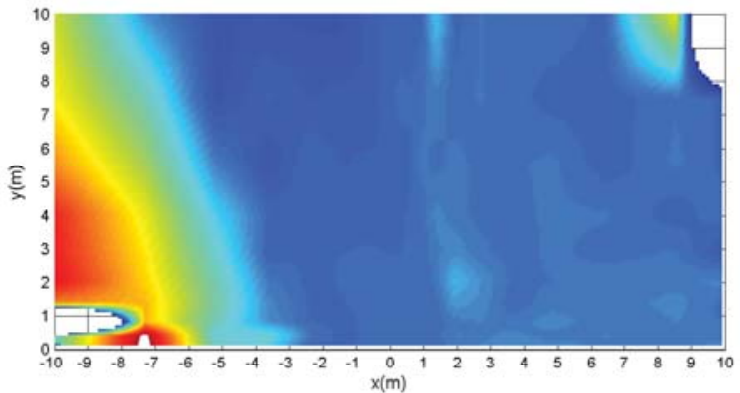

(a)

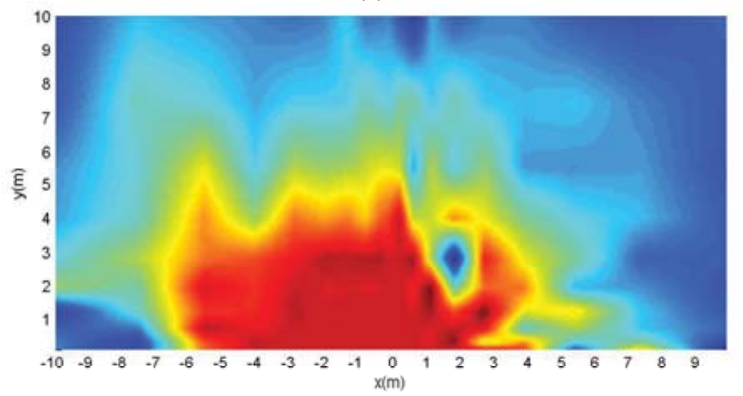

(b)

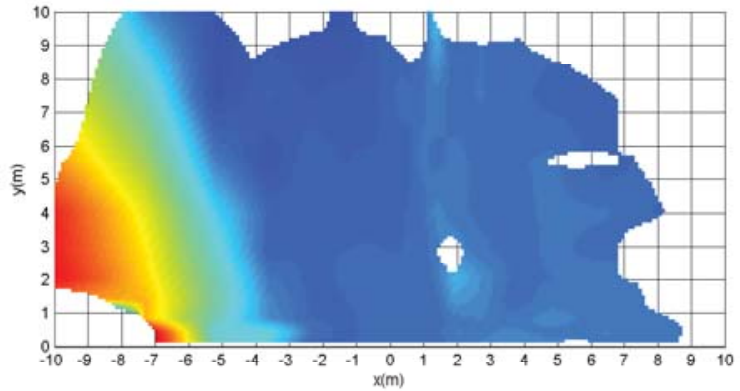

(c)

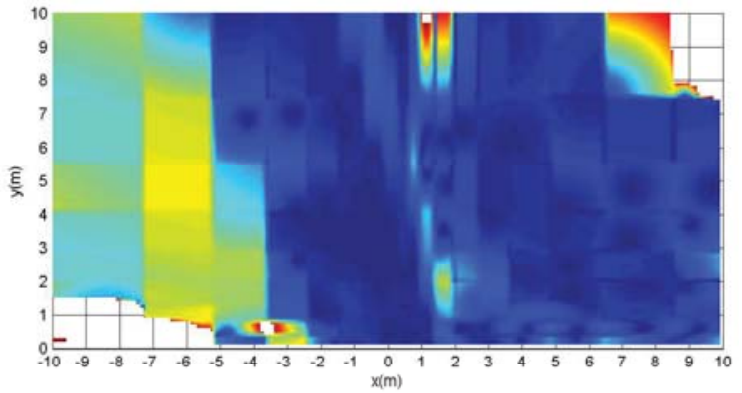

(d)

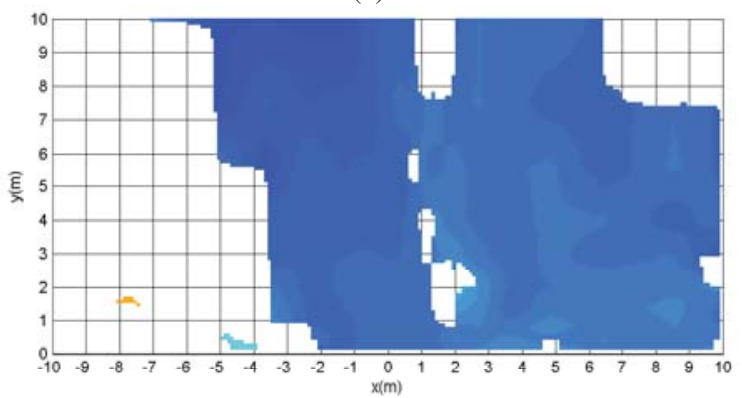

(e)

Fig. 6. Processing of the first scan: (a) Top view of the FEM, (b) fuzzy reliability mask, (c) FEM with reliability $v>0.1$, (d) FEM gradient, and (e) FEM with $|\nabla H|<0.5$. 


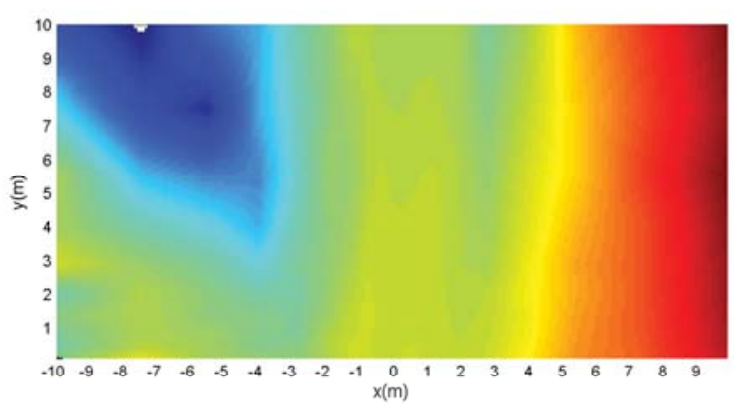

(a)

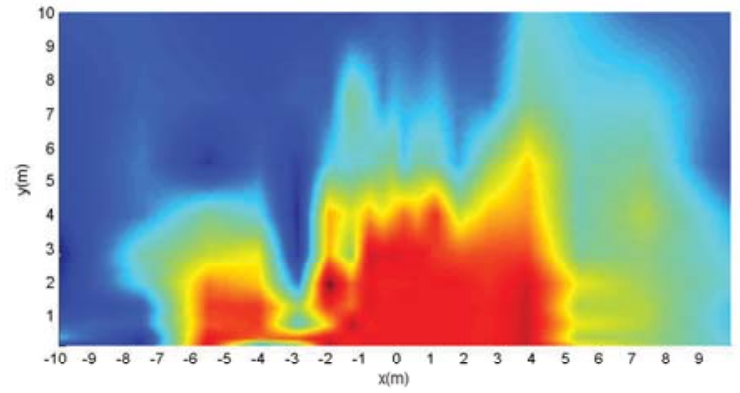

(b)

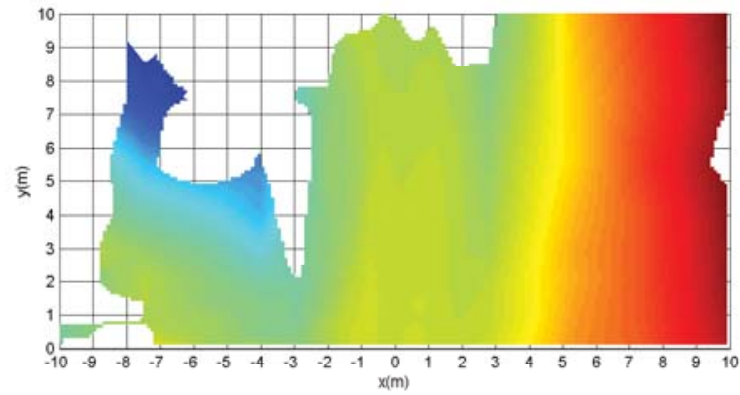

(c)

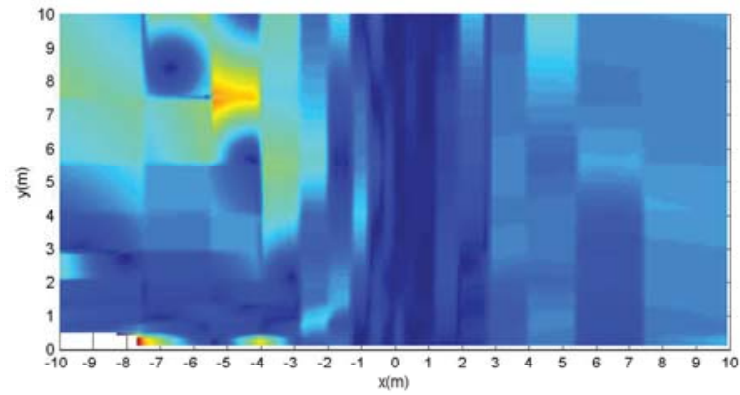

(d)

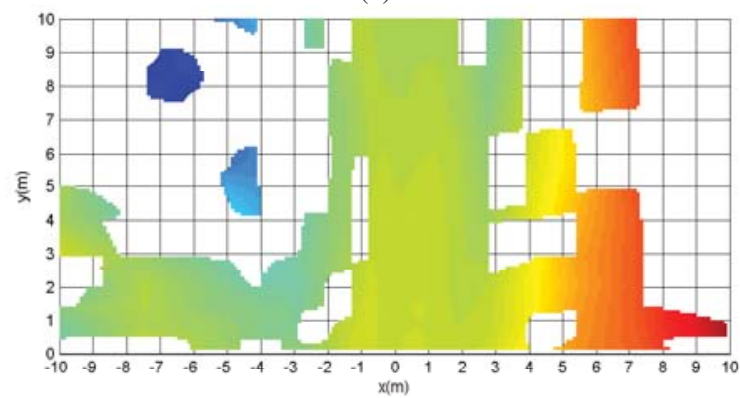

(e)

Fig. 7. Processing of the second scan: (a) Top view of the FEM, (b) fuzzy reliability mask, (c) FEM with reliability $v>0.1$, (d) FEM gradient, and (e) FEM with $|\nabla H|<0.5$.

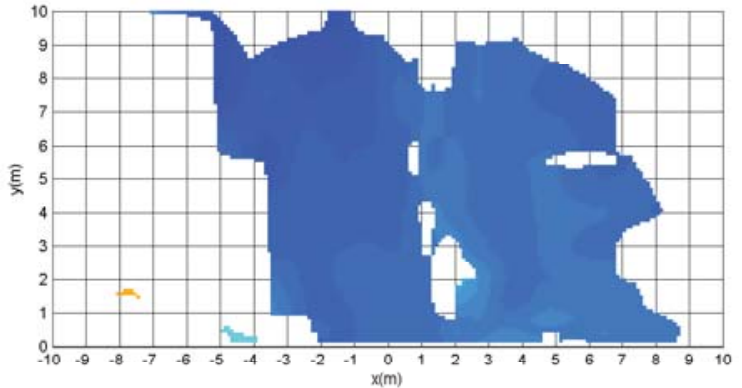

(a)

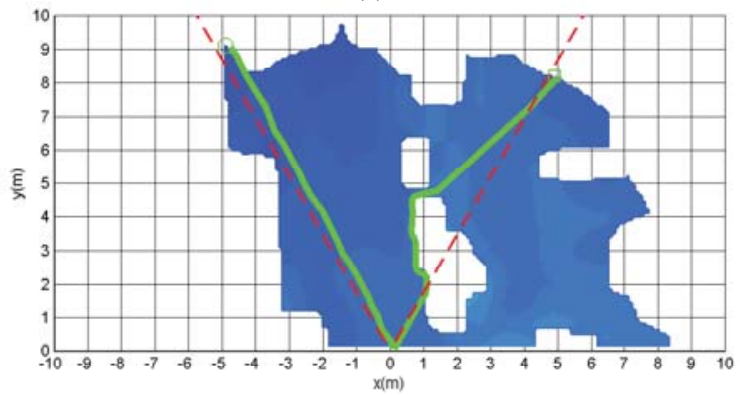

(b)

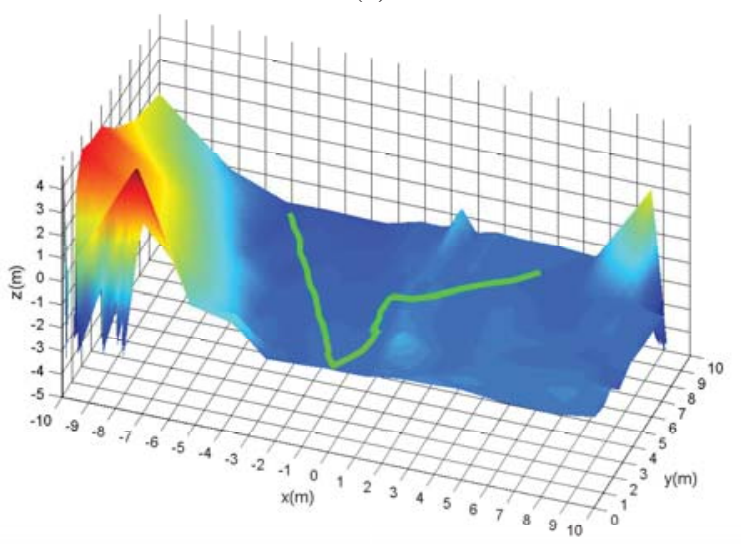

(c)

Fig. 8. First experimental site: Navigable area (a), local planned paths with two hypothetical headings for the goal points (b), and 3D view of the paths (c).

region of the FEM. This has been applied to actual 3D scans on two different experimental sites.

Future work includes navigation tests with the Quadriga mobile robot. Moreover, we are currently working on an improvement of FEM-based navigability assessment by removing overhanging objects like tree branches.

\section{ACKNOWLEDGMENTS}

This work was partially supported by the Spanish CICYT project DPI 2011-22443 and the Andalusian project PE-2010 TEP-6101.

\section{REFERENCES}

[1] D. Gingras, T. Lamarche, J. L. Bedwani, and E. Dupuis, "Rough terrain reconstruction for rover motion planning," in Canadian Conf. on Computer and Robot Vision, Ottawa, Canada, 2010, pp. 191 - 198.

[2] C. H. Tong, T. D. Barfoot, and E. Dupuis, "Three-dimensional SLAM for mapping planetary work site environments," Journal of Field Robotics, vol. 29, no. 3, pp. 381-412, 2012. 


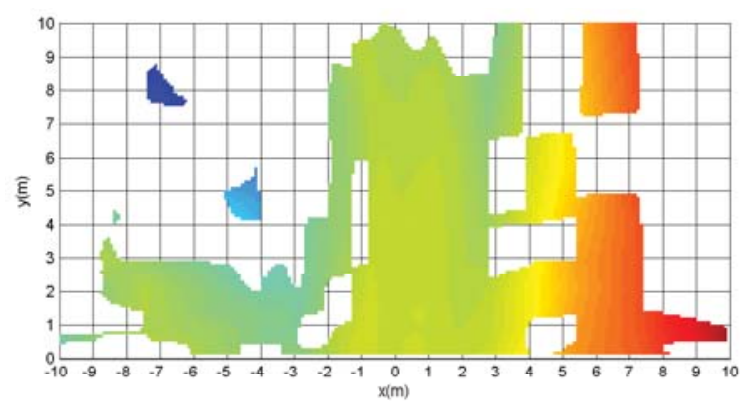

(a)

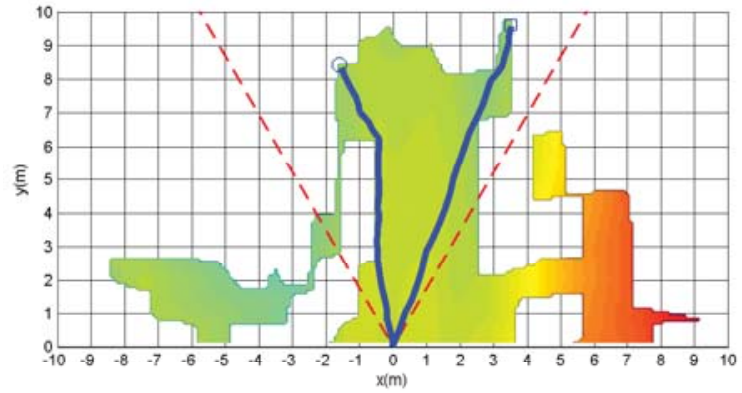

(b)

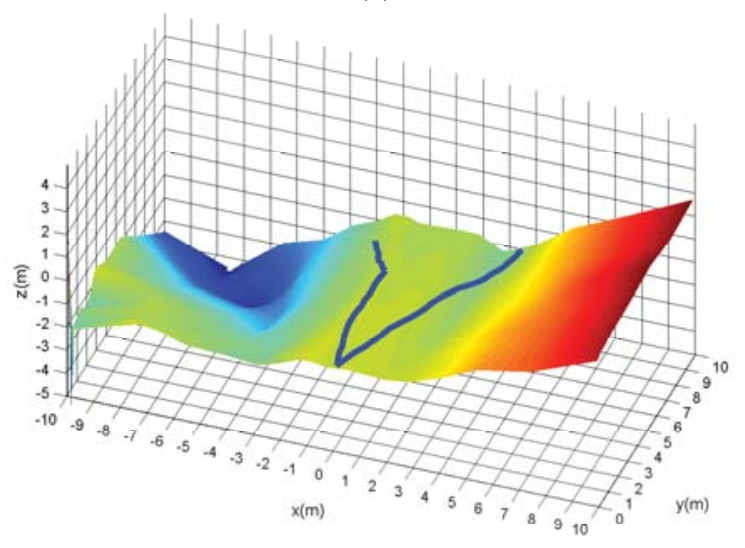

(c)

Fig. 9. Second experimental site: Navigable area (a), local planned paths with two hypothetical headings for the goal points (b), and 3D view of the paths (c).

[3] F. A. W. Belo, A. Birk, C. Brunskill, F. Kirchner, V. Lappas, C. Remy, S. Roccella, C. Rossi, A. Tikanmäki, and G. Visentin, "The ESA lunar robotics challenge: Simulating operations at the lunar south pole," Journal of Field Robotics, vol. 29, no. 4, pp. 601-626, 2012.

[4] I. Rekleitis, J. L. Bedwani, E. Dupuis, T. Lamarche, and P. Allard, "Autonomous over-the-horizon navigation using LIDAR data," $\mathrm{Au}$ tonomous Robots, vol. 34, no. 1-2, pp. 1-18, 2013.

[5] K. Nagatani, N. Tokunaga, Y. Okada, K. Yoshida, Y. Hada, T. Yoshida, and E. Koyanagi, "Teleoperation of all-terrain robot using continuous acquisition of three-dimensional environment under time-delayed narrow bandwidth communication," in IEEE Int. Workshop on Safety, Security \& Rescue Robotics, Denver, USA, 2009.

[6] A. Birk, K. Pathak, N. Vaskevicius, M. Pfingsthorn, J. Poppinga, and S. Schwertfeger, "Surface representations for 3D mapping," Künstliche Intelligenz, vol. 24, pp. 249-254, 2010.

[7] J. Bagnell, D. Bradley, D. Silver, B. Sofman, and A. Stentz, "Learning for autonomous navigation," IEEE Robotics and Automation Magazine, vol. 17, no. 2, pp. 74-84, 2010.

[8] A. M. Cretu, P. Payeur, and E. M. Petriu, "Selective range data acquisition driven by neural-gas networks," IEEE Transactions on Instrumentation and Measurement, vol. 58, no. 8, pp. 2634-2642, 2009.
[9] Y. Zhuang, N. Jiang, H. Hu, and F. Yan, "3-D-laser-based scene measurement and place recognition for mobile robots in dynamic indoor environments," IEEE Transactions on Instrumentation and Measurement, 2012.

[10] N. Vaskevicius, A. Birk, K. Pathak, and S. Schwertfeger, "Efficient representation in three-dimensional environment modeling for planetary robotic exploration," Advanced Robotics, vol. 24, no. 8-9, pp. 1169-1197, 2010.

[11] P. Pfaff, R. Triebel, and W. Burgard, "An efficient extension to elevation maps for outdoor terrain mapping and loop closing," Int. Journal of Robotics Research, vol. 26, no. 2, pp. 217-230, 2007.

[12] G. Ishigami, K. Nagatani, and K. Yoshida, "Path planning and evaluation for planetary rovers based on dynamic mobility index," in IEEE Int. Conf. on Intelligent Robots and Systems, 2011, pp. 601-606.

[13] T. Gerbaud, V. Polotski, and P. Cohen, "Simultaneous exploration and 3D mapping of unstructured environments," in IEEE Int. Conf. on Systems, Man and Cybernetics, vol. 6, 2004, pp. 5333-5337.

[14] A. Ciampalini, P. Cignoni, C. Montani, and R. Scopigno, "Multiresolution decimation based on global error," The Visual Computer, vol. 13, pp. 228-246, 1997.

[15] M. Garland and P. S. Heckbert, "Surface simplification using quadric error metrics," in ACM SIGGRAPH Conf. on Computer Graphics, 1997, pp. 209-216.

[16] J.-S. R. Jang, "ANFIS: Adaptative-network-based fuzzy inference system," IEEE Transactions on Systems, Man and Cybernetics, vol. 23, no. 3, 1993.

[17] A. Mandow, T. J. Cantador, A. García-Cerezo, A. J. Reina, J. L. Martínez, and J. Morales, "Fuzzy modeling of natural terrain elevation from a 3D scanner point cloud," in 7th IEEE Int. Symposium on Intelligent Signal Processing, Floriana, Malta, 2011.

[18] B. E. Stenning and T. D. Barfoot, "Path planning with variable-fidelity terrain assessment," Robotics and Autonomous Systems, vol. 60, no. 9, pp. 1135-1148, 2012.

[19] C. Castejón, D. Blanco, and L. Moreno, "Compact modeling technique for outdoor navigation," IEEE Transactions on Systems, Man, and Cybernetics Part A: Systems and Humans, vol. 38, no. 1, pp. 9-24, 2008.

[20] D. Gingras, E. Dupuis, G. Payre, and J. De Lafontaine, "Path planning based on fluid mechanics for mobile robots using unstructured terrain models," in IEEE Int. Conf. on Robotics and Automation, Anchorage, USA, 2010, pp. 1978-1984.

[21] S. Lacroix, A. Mallet, D. Bonnafous, G. Bauzil, S. Fleury, M. Herrb, and R. Chatila, "Autonomous rover navigation on unknown terrains: functions and integration," in The Int. Journal of Robotics Research, no. 21, 2002, pp. $917-942$.

[22] Z.-H. Xiu and G. Ren, "Stability analysis and systematic design of Takagi-Sugeno fuzzy control systems," Fuzzy Sets and Systems, vol. 151, no. 1, pp. $119-138,2005$.

[23] V. J. Lumelsky and A. A. Stepanov, "Path-planning strategies for a point mobile automaton moving amidst unknown obstacles of arbitrary shape," Algorithmica, vol. 2, no. 1, pp. 403-430, 1987.

[24] H. Choset, K. M. Lynch, S. Hutchinson, G. A. Kantor, W. Burgard, L. E. Kavraki, and S. Thrun, Principles of Robot Motion: Theory, Algorithms, and Implementations. Cambridge, USA: MIT Press, 2005.

[25] B. M. Leedy, J. S. Putney, C. Bauman, S. Cacciola, J. M. Webster, and C. F. Reinholtz, "Virginia Tech's twin contenders: A comparative study of reactive and deliberative navigation," Journal of Field Robotics, vol. 23, no. 9, pp. 709-727, 2006.

[26] J. Morales, J. L. Martínez, A. Mandow, A. García-Cerezo, and S. Pedraza, "Power consumption modeling of skid-steer tracked mobile robots on rigid terrain," IEEE Transactions on Robotics, vol. 25, no. 5, pp. 1098-1108, 2009.

[27] J. Morales, J. L. Martínez, A. Mandow, A. Pequeño-Boter, and A. García-Cerezo, "Design and development of a fast and precise low-cost 3D laser rangefinder," in IEEE Int. Conf. on Mechatronics, Istanbul, Turkey, 2011, pp. $621-626$.

[28] J. Morales, J. L. Martínez, A. Mandow, A. Pequeño-Boter, and A. García-Cerezo, "Simplified power consumption modeling and identification for wheeled skid-steer robotic vehicles on hard horizontal ground," in IEEE/RSJ Int. Conf. on Intelligent Robots and Systems, Taipei, Taiwan, 2010, pp. 4769-4774. 